\title{
Reviewers for the Special Issue on Contemporary Youth Sport: Critical Issues and Future Directions
}

\author{
Anthony Amorose, Illinois State University, USA \\ Lindsey Blom, Ball State University, USA \\ Ian Boardley, University of Birmingham, UK \\ Steven Bray, McMaster University, Canada \\ Mark Bruner, Nipissing University, Canada \\ Travis Dorsch, Utah State University, USA \\ Andy Driska, Michigan State University, USA \\ Robert Elbin, University of Arkansas, USA \\ Nick Holt, University of Alberta, Canada \\ Thelma Horn, Miami University, USA \\ Anthony Kontos, University of Pittsburgh, USA
}

Leapetswe Malete, Michigan State University, USA Eric Martin, Boise State University, USA Jennifer Nalepa, Michigan State University, USA Karin Pfeiffer, Michigan State University, USA

Scott Pierce, Illinois State University, USA Mustafa Sarkar, Nottingham Trent University, UK Deborah Shapiro, Georgia State University, USA Shannon Siegel, University of San Francisco, USA Dana Voelker, West Virginia University, USA Maureen Weiss, University of Minnesota, USA Meredith Whitley, Adelphi University, USA 